\section{Revisiting the link between platelets and depres- sion through genetic epidemiology: new insights from platelet distribution width}

For many years, the link between platelets and depression (or major depressive disorder, MDD) has been a matter of investigation. Indeed, platelets share numerous features with neurons ${ }^{1}$ and represent the first reservoir of serotonin - one of the key neurotransmitters in the pathophysiology of MDD - in the human body. ${ }^{2}$ Several epidemiological studies investigated the relation between $\mathrm{MDD}$ and platelet parameters, such as mean platelet volume (MPV) and platelet count (Plt). Canan and colleagues ${ }^{3}$ observed a positive association between MPV and MDD in a Turkish population (287 cases and 1,999 controls), which was significant in females, but not in males. This association was later replicated in a case-control setting (103 MDD patients and 106 controls) ${ }^{4}$ and in a hospital-based study (90 cases and 49 controls), ${ }^{5}$ although no analysis stratified by sex was performed in these studies. Regarding platelet count, contrasting evidence of association with MDD status has been reported..$^{3-5}$ A positive association with plateletcrit i.e., the product of MPV and platelet count - was also found. ${ }^{4}$ In a small study comparing 31 patients with lifelong recurrent depression treated with selective serotonin reuptake inhibitors and 31 matched healthy controls, Aleksovski et al. ${ }^{6}$ reported significantly greater MPV, platelet distribution width (PDW) and platelet-tolarger cell ratio for depressed participants. In these patients, lower platelet and blood plasma serotonin levels were also observed, along with lower platelet reactivity, as suggested by collagen- and epinephrine-induced aggregometry, and by the percentage of spiny and discoid platelets. ${ }^{6}$ More recently, our group identified a significant positive association between depressive symptoms and PDW, in a study analyzing the relation between low-grade inflammation and mental health in a large Italian population cohort $(\mathrm{N}=12,732)$. This survived a conservative correction for multiple testing and adjustment for several sociodemographic, health and lifestyle covariates, suggesting partly shared genetic bases between depression risk and platelet traits.'

These findings prompted us to re-visit the link between platelets and MDD, investigating whether the significant associations observed between platelet indices and MDD in previous epidemiological studies could be explained by common genetic factors - in particular single nucleotide polymorphisms and small insertions/deletions (indels) - underlying both depression and

Table 1. Genetic correlations ( $r g$ ) computed in linkage disequilibrium score regression analysis between risk of major depressive disorder $^{10}$ and three platelet parameters. ${ }^{11}$

\begin{tabular}{lccccc}
$\begin{array}{l}\text { Platelet } \\
\text { parameter }\end{array}$ & \#SNPa & $\mathrm{Ig}_{\mathrm{g}}$ & SE & Z-score & $\boldsymbol{P}$ \\
Plt & $1,087,093$ & -0.004 & 0.028 & -0.14 & 0.89 \\
MPV & $1,087,076$ & 0.041 & 0.029 & 1.41 & 0.16 \\
\hline PDW & $1,086,805$ & 0.079 & 0.029 & 2.75 & 0.006
\end{tabular}

The three platelet parameters analyzed were platelet count, mean platelet volume, and platelet distribution width. Significant genetic correlations surviving the Bonferroni correction $(P<0.017)$ are reported in bold. ${ }^{a}$ Number of variants actually used in the linkage disequilibrium score regression after quality control filters (see Online Supplementary Methods for details). SNP: single nucleotide polymorphism; SE: standard error; MPV: mean platelet volume; PDW: platelet distribution width. variability in platelet parameters. We did this by computing cross-trait genetic correlations through linkage disequilibrium (LD) score regression analysis, ${ }^{8,9}$ using the summary statistics of large, independent genome wide association studies (GWAS) previously conducted on depression $(59,851 \text { cases and } 113,154 \text { controls })^{10}$ and platelet parameters $\left(\mathrm{N}_{\max }=166,066\right)^{11}$ (see Online Supplementary Methods and Online Supplementary Table S1 for details). Although this analysis has already been performed for Plt and $\mathrm{MPV},{ }^{10}$ here we also analyzed PDW, which reflects individual variation and heterogeneity of platelet size. Moreover, we used GWAS summary statistics of platelet parameters from a much larger sample $\left(\mathrm{N}_{\max } \sim 166,000\right)^{11}$ than the one used before $(\mathrm{N} \sim 67,000) .{ }^{12}$ This analysis revealed a significant genetic correlation between PDW and MDD risk $\left[\mathrm{r}_{\mathrm{g}}=0.079\right.$ standard error $(\mathrm{SE})=0.029 ; P=0.006)$, which survived the Bonferroni correction for the three platelet parameters tested ( $\alpha=0.017$ ) (Table 1$)$. No significant genetic correlation was observed for MPV and Plt, in line with previous analyses of these parameters. ${ }^{10}$

To disentangle causal relationships between PDW and MDD, we used GWAS summary statistics to carry out a two-sample Mendelian randomization (MR) analysis, through the TwoSampleMR package in $\mathrm{R} .{ }^{13}$ We used inverse variance weighted regressions to model the relation between single nucleotide polymorphism-exposure and -outcome effects for each of the genetic (instrumental) variants analyzed (see the Online Supplementary Methods for details). Under the hypothesis of a bi-directional causality link, we modeled MR regressions assuming PDW as the exposure and MDD as the outcome, and vice versa, using strictly $\mathrm{LD}$-pruned independent variants which showed genome-wide significant associations in the exposure study $\left(P<5 \times 10^{-8}\right)$. This analysis did not reveal any evidence of causality between PDW and $\mathrm{MDD}$, either when assuming PDW $[\beta(\mathrm{SE})=-0.004$ (0.019), $P=0.83$; over 114 instrumental variants], or

Table 2. Results of the Mendelian randomization analysis, assuming (A) platelet distribution width (PDW) as the exposure variable and depression (MDD) as the outcome (PDW $\rightarrow$ MDD); and (B) vice versa (MDD $\rightarrow$ PDW).

\begin{tabular}{lcccc} 
(A) & & & & \\
Method & \#SNPa & $\beta$ & SE & $\boldsymbol{P}$ \\
MR Egger & 114 & -0.036 & 0.037 & 0.33 \\
\hline Weighted median & 114 & -0.023 & 0.031 & 0.46 \\
IVW & 114 & -0.004 & 0.019 & 0.83 \\
\hline Simple mode & 114 & 0.022 & 0.058 & 0.71 \\
Weighted mode & 114 & -0.018 & 0.038 & 0.64 \\
\hline
\end{tabular}

\begin{tabular}{lcccc} 
(B) & & & & \\
Method & \#SN ${ }^{\mathrm{a}}$ & $\boldsymbol{\beta}$ & SE & $\boldsymbol{P}$ \\
MR Egger & 4 & -0.021 & 0.194 & 0.93 \\
\hline Weighted median & 4 & -0.018 & 0.044 & 0.69 \\
IVW & 4 & -0.015 & 0.038 & 0.68 \\
\hline Simple mode & 4 & -0.019 & 0.064 & 0.79 \\
Weighted mode & 4 & -0.02 & 0.065 & 0.78 \\
\hline
\end{tabular}

Here, the results of different Mendelian randomization (MR) methods as per TwoSampleMR output are reported (see Hemani et al. ${ }^{13}$ for details), for completeness of information. SNP: single nucleotide polymorphisms; SE: standard error; MR Egger: Mendelian randomization, Egger method; IVW: inverse variance weighted

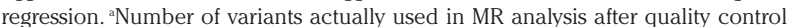
filters (see Online Supplementary Methods for details) 
when assuming MDD status as exposure $[\beta(\mathrm{SE})=-0.015$ (0.038), $P=0.68$; over 4 variants]. These data were confirmed when using Egger regression (Table 2A, B), which is more robust to unbalanced pleiotropy of instrumental variants (see Online Supplementary Methods for details). ${ }^{13,14}$ A similar analysis on platelet parameters and MDD risk had already been performed in a previous GWAS, ${ }^{11}$ using association statistics from a notably smaller genetic study on depression $(9,240 \mathrm{MDD}$ cases and 9,519 controls) and investigating only causal effects of platelet parameters on MDD risk. ${ }^{11}$ This analysis revealed no evidence of significant causal links, in line with our findings, although the authors reported marginally significant effects, which did not survive correction for multiple testing, for MPV and PDW. ${ }^{11}$ Although our MR results may appear to be in contrast with the significant genetic correlation identified above, it is worth underlining that here LD score regression was performed over more than one million variants genome-wide, while MR was carried out on around 100 variants, at most. Therefore, the significant genetic correlation observed between PDW and MDD risk is more robust than and not truly comparable with the lack of evidence of a causal effect between these phenotypes, which may be due to a lack of power of our MR analysis. Similarly, the two studies employed here ${ }^{10,11}$ did not include replication samples, hence some of the genome-wide significant variants (i.e. the instrumental variants used in MR) may be false positive and affect the results of the MR analysis. As the sample size of genetic studies becomes larger and larger, and more genetic variants influencing the traits are discovered, we will have more powerful means to better disentangle the molecular architecture of depression and the nature of its link with platelets, through genetic epidemiology approaches. Another possible explanation for the discrepancy between LD score regression and $M R$ analysis is that the latter assumes non-pleiotropy of the instrumental variants, and even methods such as Egger regression may not account completely for complex pleiotropic relationships which may occur between the instrumental variants used, PDW and MDD risk. Another limitation of our work is the lack of genetic analyses (hence of summary statistics) stratified by sex in the original GWAS, ${ }^{10,11}$ which did not allow us to check whether differential genetic relationships occur between PDW and MDD risk based on sex. Indeed, in these studies both platelet parameters and MDD risk were analyzed including sex among covariates, ${ }^{10,11}$ which in some cases may lead to different results, compared to stratifying genetic associations by sex.

In spite of these limitations, our findings point towards a new platelet parameter, PDW, which so far has been fairly neglected in neuropsychiatric research. Besides the association with depressive symptoms identified by our group, ${ }^{7}$ an increased PDW has recently been reported to be present in patients affected by recurrent depression resistant to treatment with selective serotonin reuptake inhibitors ${ }^{6}$ and by panic disorder, a neuropsychiatric condition which is thought to have shared biological bases with MDD. ${ }^{15}$ This suggests that PDW is implicated - either directly or indirectly - in the neurobiology of depression and comorbid disorders. However, the significance of this parameter in relation to platelet function in non-pathological settings (i.e., in general population studies) remains largely unknown. We can speculate that, as an index expressing heterogeneity of platelet size and in light of previous associations reported with indices of platelet activation, ${ }^{16}$ PDW might be a useful marker of platelet function, as previously suggest- ed for MPV. ${ }^{17-19}$ Although more and larger studies in nonpathological settings are needed to confirm this concept, this suggests a link between PDW and platelet function in the context of activation of the hemostatic system, which may potentially extend to other domains, such as cardiovascular and neuropsychiatric domains.

Overall, the evidence reported here supports PDW as a new, potential biomarker of depression and psychopathology. Further investigations of this parameter in epidemiological, genetic and molecular studies are warranted.

Alessandro Gialluisi, ${ }^{1}$ Benedetta Izzi, ${ }^{1}$ Augusto Di

Castelnuovo, ${ }^{2}$ Chiara Cerletti, ${ }^{1}$ Maria Bendetta Donati, Giovanni de Gaetano ${ }^{1}$ and Licia Iacoviello,

'Department of Epidemiology and Prevention, IRCCS Neuromed, Pozzilli; ${ }^{2}$ Mediterranea Cardiocentro, Napoli and ${ }^{3}$ Department of Medicine and Surgery, University of Insubria, Varese, Italy

Correspondence: ALESSANDRO GIALLUISI

alessandro.gialluisi@gmail.com

\section{doi:10.3324/haematol.2019.222513}

Acknowledgments: the present analyses were partially supported by the Italian Association for Cancer Research (AIRC) with grant AIRC' $5 \times 1000$ ' to LI (reference number 12237). BI was a postdoctoral fellow of the Fondazione Umberto Veronesi, Milan, Italy. We are grateful to Prof Marc Hoylaerts for his informal review of the manuscript.

Information on authorship, contributions, and financial \& other disclosures was provided by the authors and is available with the online version of this article at www. haematologica.org.

\section{References}

1. de Gaetano G. Blood platelets as a pharmacological model of serotoninergic synaptosomes. In: de Gaetano G, and Garattini S, editors. Platelets: A Multidisciplinary Approach. New York: Raven Press. 1978. p373-384.

2. Williams MS. Platelets and depression in cardiovascular disease: a brief review of the current literature. World J Psychiatry. 2012;2(6):114-123

3. Canan F, Dikici S, Kutlucan A, et al. Association of mean platelet volume with DSM-IV major depression in a large communitybased population: the MELEN study. J Psychiatr Res. 2012;46(3):298-302.

4. Cai L, Xu L, Wei L, Chen W. Relationship of mean platelet volume to MDD: a retrospective study. Shangai Arch Psychiatry. 2017;29(1):21-29

5. Bondade S, Supriya, Seema HS, Shivakumar BK. Mean platelet volume in depression and anxiety disorder- a hospital based casecontrol study. Int Neuropsychiatr Dis J. 2018;11(4):1-8.

6. Aleksovski B, Neceva V, Vujović V, et al. SSRI-reduced platelet reactivity in non-responding patients with life-long recurrent depressive disorder: detection and involved mechanisms. Thromb Res. 2018;165:24-32

7. Gialluisi A, Bonaccio M, Di Castelnuovo A, et al. Lifestyle and biological factors influence the relationship between mental health and low-grade inflammation. Brain Behav Immun. 2019 May 2. [Epub Ahead of Print]

8. Bulik-Sullivan B, Finucane HK, Anttila V, et al. An atlas of genetic correlations across human diseases and traits. Nat Genet. 2015;47(11):1236-1241

9. Bulik-Sullivan B, Loh PR, Finucane HK, et al. LD score regression distinguishes confounding from polygenicity in genome-wide association studies. Nat Genet. 2015;47(3):291-295.

10. Wray NR, Ripke S, Mattheisen M, et al. Genome-wide association analyses identify 44 risk variants and refine the genetic architecture of major depression. Nat Genet. 2018;50(5):668-681.

11. Astle WJ, Elding H, Jiang $T$, et al. The allelic landscape of human blood cell trait variation and links to common complex disease. Cell. 2016;167(5):1415-1429.

12. Gieger C, Radhakrishnan A, Cvejic A, et al. New gene functions in megakaryopoiesis and platelet formation. Nature. 


\section{LETTERS TO THE EDITOR}

2011;480(7376):201-208.

13. Hemani G, Zheng J, Elsworth B, et al. The MR-base platform supports systematic causal inference across the human phenome. Elife. 2018;7.

14. Burgess S, Thompson SG. Interpreting findings from Mendelian randomization using the MR-Egger method. Eur J Epidemiol. 2017;32(5):377-389.

15. Ransing RS, Patil B, Grigo O. Mean platelet volume and platelet distribution width level in patients with panic disorder. J Neurosci Rural Pract. 2017;8(2):174-178.

16. Vagdatli E, Gounari E, Lazaridou E, Katsibourlia E, Tsikopoulou F, Labrianou I. Platelet distribution width: a simple, practical and specific marker of activation of coagulation. Hippokratia. 2010;14(1):28-32.

17. Ntolios P, Papanas N, Nena E, et al. Mean platelet volume as a surrogate marker for platelet activation in patients with idiopathic pulmonary fibrosis. Clin Appl Thromb. 2016;22(4):346-350.

18. Braekkan SK, Mathiesen EB, Njolstad I, Wilsgaard T, Stormer J, Hansen JB. Mean platelet volume is a risk factor for venous thromboembolism: the Tromsø study. J Thromb Haemost. 2010;8(1):157-162.

19. Chu SG, Becker RC, Berger PB, et al. Mean platelet volume as a predictor of cardiovascular risk: a systematic review and metaanalysis. J Thromb Haemost. 2010;8(1):148-156. 